\title{
Transdiagnostic Therapy in the Treatment of Eating Disorders
}

\section{Background/Aims}

Cognitive-behavioral therapy - psychotherapeutic technique with aim in changing maladaptive cognitions and related affects and behaviors; - Significative therapeutic effect on multiple psychiatric pathology - including eating disorders $-+/$ - medication;

Eating disorders - persistent pattern of abnormal feeding behavior $+/$ - abnormal food intake + negative effects for physical, psychological and social areas of the individual;

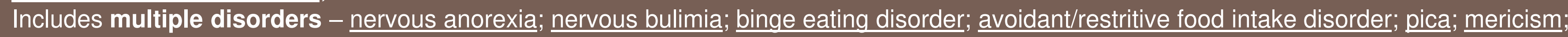
Transdiagnostic Therapy directs the treatment to the common psychopatological basis of these multiple disorders.

\section{Objectives}

With this work authors want to review the basis of transdiagnostic psychotherapeutic approach in the context of eating disorders.

\section{Materials and methods}

The authors reviewed, non systematically, the literature, through search in textbooks and papers related to this topic in the databases of Pubmed and Sciencedirect.

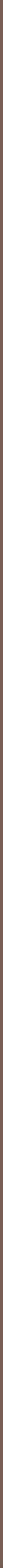

